JOURNAL OF THE

AMERICAN MATHEMATICAL SOCIETY

Volume 17, Number 2, Pages 361-372

S 0894-0347(03)00448-X

Article electronically published on November 25, 2003

\title{
PLURISUBHARMONIC DOMINATION
}

\author{
LÁSZLÓ LEMPERT
}

\section{INTRODUCTION}

A common device in several complex variables, notably in the theory of Stein spaces, is to exhaust a space $S$ by a sequence of holomorphically convex compact subsets $K_{j}$. Analytical problems one has to solve on $S$ (say, a Cousin problem) tend to become more manageable when restricted to $K_{j}$ because over compact sets the data is uniformly controlled; on the other hand, once the problem is solved on $K_{j}$, it is often possible to pass to a solution on $S=\bigcup K_{j}$.

In complex analysis on infinite dimensional spaces compact exhaustions are not available: no open set in an infinite dimensional Banach space (other than $\emptyset$ ) can be represented as a countable union of compact sets. This paper is about a device in certain Banach spaces that makes up for the lack of compact exhaustions.

Recall that an infinite dimensional Banach space $X$ (always over $\mathbb{C}$ ) has a Schauder basis $e_{1}, e_{2}, \ldots \in X$ if any $x \in X$ can be uniquely represented as a norm convergent sum

$$
x=\sum \lambda_{n} e_{n}, \quad \lambda_{n} \in \mathbb{C} .
$$

If in addition the series in (1.1) converge after arbitrary rearrangements, one speaks of a countable unconditional basis. For example the spaces $l^{p}, L^{p}[0,1], 1<p<\infty$, $l^{1}$, and $c$ (the space of convergent sequences) have an unconditional basis, but $L^{1}[0,1]$ and $C[0,1]$ only have a Schauder basis; see $[\mathbf{S n}$. Recall further that an open $\Omega \subset X$ is pseudoconvex if $\Omega \cap Y$ is such for all finite dimensional subspaces $Y \subset X$. Similarly, an upper semicontinuous $v: \Omega \rightarrow[-\infty, \infty)$ is plurisubharmonic if $v \mid Y$ is, for all finite dimensional $Y \subset X$. Our main result is

Theorem 1.1. Suppose $X$ is a Banach space with countable unconditional basis, $\Omega \subset X$ open and pseudoconvex, $u: \Omega \rightarrow \mathbb{R}$ locally bounded above. There is a plurisubharmonic $v: \Omega \rightarrow \mathbb{R}$ such that $u(x) \leq v(x)$ for $x \in \Omega$. Even better, there are a Banach space $\left(V,\|\|_{V}\right)$ and a holomorphic $f: \Omega \rightarrow V$ such that $u(x) \leq\|f(x)\|_{V}$ for $x \in \Omega$.

We shall concisely refer to the statements of the theorem by saying that in $\Omega$ plurisubharmonic, resp. holomorphic, domination is possible.

It is not hard to see that the Banach space $V$ of the theorem can be chosen separable. Once this is known, one can also choose it to be $l^{\infty}$ or $C[0,1]$, since an arbitrary separable Banach space embeds in $l^{\infty}$ and in $C[0,1]$. An important

Received by the editors March 6, 2003.

2000 Mathematics Subject Classification. Primary 32Txx, 32U05, 46G20.

Research partially supported by an NSF grant. 
question arises - especially in connection with Theorem 1.3 below and its proofwhether $V$ can be taken to have an unconditional basis or, if $X$ is a Hilbert space, if $V$ itself can be taken to be a Hilbert space. We do not know the answer.

The theorem can be used as follows. Suppose we are given a complex analytical object in $\Omega$, for example, a Cousin problem. One can control the size of the object at each $x \in \Omega$ by a function $u(x)$; typically $u$ will be locally bounded. With $v$ as in the theorem and $\Omega_{j}=\{x \in \Omega: v(x)<j\}, j \in \mathbb{N}$, the object is then under uniform control on each pseudoconvex set $\Omega_{j}$, just as if $\Omega_{j}$ were compact. For this reason it is natural to expect that on $\Omega_{j}$ the object is easier to study; and, if this is indeed so, one may eventually be able to understand it on all of $\Omega=\bigcup \Omega_{j}$. We shall use a variant of this idea to study bundle valued analytic cohomology groups in [L4], and here we restrict ourselves to three simpler applications. The first really just reformulates plurisubharmonic domination in geometric terms:

Theorem 1.2. Let $X, Y$ be Banach spaces, $\Omega \subset X$ open and pseudoconvex. If plurisubharmonic domination is possible in $\Omega$, then $\Omega \times\{0\} \subset X \times Y$ has a neighborhood basis consisting of pseudoconvex sets.

This is related to Siu's theorem on Stein neighborhoods of Stein subvarieties of finite dimensional complex spaces in $[\mathrm{Su}]$ and can be thought of as a first step in generalizing that theorem to infinite dimensions. Of course, both Siu's theorem and Theorem 1.2 revolve around constructing plurisubharmonic functions, but the difficulties are different: there the difficulty is the nonlinear nature of the geometry involved, here it is the lack of compact exhaustions.

The second application is an infinite dimensional version of the BishopNarasimhan-Remmert embedding theorem $\mathrm{B}, \mathrm{Na}, \mathrm{R}$ :

Theorem 1.3. If $X$ is a Banach space and if in an open $\Omega \subset X$ holomorphic domination is possible, then $\Omega$ is biholomorphic to a closed, rectifiable, and split complex submanifold of a Banach space.

That a submanifold $N$ of a complex manifold $M$ is rectifiable means that the pair $(M, N)$ is locally biholomorphic to a pair $(Y, Z), Y$ a Banach space and $Z$ a closed subspace. For this notion see [L1, Section 2]. If, in addition, the local model $Z \subset Y$ has a closed complement, one says $N$ is split. A related result is proved in Arroud's thesis: if a Banach space $X$ has a Schauder basis, then any pseudoconvex $\Omega \subset X$ can be properly embedded in some locally convex space [A Théorème 2.1].

We will have to introduce a few concepts before presenting the third application. If $M$ is a complex manifold and $E \rightarrow M$ is a Banach bundle, a metric on $E$ is a function $p: E \rightarrow[0, \infty)$ that restricts on each fiber to a norm inducing the topology of the fiber. If $E$ is a holomorphic vector bundle and $p$ is plurisubharmonic, we shall also say that $p$ and $E$ are seminegatively curved, or just seminegative. A metric $p$ on $E$ determines a metric $p^{*}$ on the dual bundle $E^{*}$; if the latter is seminegative, we shall say $p$ is semipositive.

Theorem 1.4. Let $X$ be a separable Banach space, let $\Omega \subset X$ be open, and let $E \rightarrow \Omega$ be a locally trivial holomorphic Banach bundle. If in $\Omega$ plurisubharmonic domination is possible, then $E$ admits both a seminegative metric $p$ and a semipositive metric $q$. Given a locally bounded metric $m$ on $E, p$ can be chosen to satisfy $p \geq m$; if the dual metric $m^{*}$ rather than $m$ is locally bounded, $q$ can be chosen to satisfy $q \leq m$. If in $\Omega$ holomorphic domination is possible, $p$ and $q$ can be chosen locally Lipschitz continuous. 
Per Lipschitz continuity: on a metric space one can speak of global and local Lipschitz continuity of functions. For functions defined on a manifold, global Lipschitz continuity makes no sense, but local Lipschitz continuity does, since locally the manifold is identified with a metric space, and two local identifications are locally Lipschitz continuous with respect to one another.

It is interesting to note that, in spite of the analogy between plurisubharmonic and convex functions, in Theorem $1.1 v$ cannot be taken convex and continuous, even if $\Omega$ itself is convex. This was pointed out by Mazet in $\mathrm{Ma}$. For example, any $v: X \rightarrow \mathbb{R}$ convex and continuous will be bounded above on some ball of radius $2 \epsilon>0$, whence convexity forces $v$ to be bounded above on all balls of radius $\epsilon$. However, as soon as $\operatorname{dim} X=\infty$, there is a locally bounded $u: X \rightarrow \mathbb{R}$ that is unbounded above on a sequence of balls of radius $\rightarrow 0$; this $u$ therefore cannot be dominated by $v$.

As to the assumptions of Theorem 1.1, pseudoconvexity of $\Omega$ is clearly necessary, and some condition is also needed on $X$. Indeed, according to Dineen, the space $X=l^{\infty}$ contains infinite discrete sets on which every holomorphic function must be bounded; see [D] and also [J1. This implies that in $l^{\infty}$ holomorphic domination is impossible. We shall derive Theorem 1.1 from a more general result though, in which $X$ is assumed to have a Schauder basis and a property that involves Runge approximation. Let \|\| denote the norm in $X, B(x, r)=\{z \in X:\|x-z\|<r\}$, $B(0, r)=B(r)$; if $\Omega \subset X$ is open and $V$ is a Banach space, let $\mathcal{O}(\Omega ; V)$ stand for the space of holomorphic maps $\Omega \rightarrow V$, and $\mathcal{O}(\Omega)=\mathcal{O}(\Omega ; \mathbb{C})$. Similarly to [L3] we introduce the following

Hypothesis 1.5. There is a $\mu \in(0,1)$ such that for any Banach space $\left(W,\|\|_{W}\right)$, $\epsilon>0$, and $g \in \mathcal{O}(B(1) ; W)$ there is an $h \in \mathcal{O}(X ; W)$ that satisfies $\|g-h\|_{W}<\epsilon$ on $B(\mu)$.

Theorem 1.6. If a Banach space $X$ has a Schauder basis and satisfies Hypothesis 1.5, then holomorphic domination is possible in any pseudoconvex open $\Omega \subset X$.

In L2 we prove that Hypothesis 1.5 holds true if $X$ has a countable unconditional basis - a different proof was given by Josefson in [J2] - so Theorem 1.6 implies Theorem 1.1. The hypothesis also holds for Banach spaces that arise as $l^{1}$ sums of countably many finite dimensional spaces, as shown in $[\mathrm{P}]$.

Plurisubharmonic domination is about passing from local to global and is distantly reminiscent of cohomological problems, except that it is cruder than, say, resolving an analytical cocycle. Still, our proof here is inspired by the approach to the cohomology vanishing theorem in [L3]. It will be presented in Section 2, with auxiliary material postponed to Sections 3 and 4. Interestingly, the dominating function never gets constructed; instead we show that the assumption that a dominating function does not exist leads to a contradiction. Theorems 1.2, 1.3, and 1.4 will be proved in Section 5.

For basic complex analysis and geometry in finite and infinite dimensional spaces that we are going to rely on in this paper, the reader is referred to $[\mathrm{H}, \mathrm{L1}, \mathrm{Mu}, \overline{\mathrm{No}}$.

\section{The Proof of Theorem 1.6}

Loosely speaking, this proof will be by induction, more exactly: by infinite descent, on the size of those subsets of $\Omega$ on which $u$ can be dominated holomorphically. The technically demanding part of the proof is the "induction step", 
Proposition 2.1 below, whose proof we postpone until Section 4 . We start by setting up notation.

Let $e_{1}, e_{2}, \ldots \in X$ be a Schauder basis, and introduce the projections $\pi_{N}: X \rightarrow$ $X$,

$$
\pi_{N} \sum_{1}^{\infty} \lambda_{j} e_{j}=\sum_{1}^{N} \lambda_{j} e_{j}, \quad \lambda_{j} \in \mathbb{C} ; \quad \pi_{0}=0, \pi_{\infty}=\mathrm{id} .
$$

We shall assume for all $x \in X$

$$
\left\|\pi_{N} x-\pi_{M} x\right\| \leq\left\|\pi_{n} x-\pi_{m} x\right\|, \quad 0 \leq n \leq N \leq M \leq m \leq \infty .
$$

As pointed out in [L3, Section 7], this can always be arranged upon equivalently renorming $X$. Consider the covering $\mathfrak{B}$ of $\Omega \subset X$

$$
\mathfrak{B}=\mathfrak{B}_{\Omega}=\{\text { balls } B: \bar{B} \subset \Omega, 2 \operatorname{diam} B<\operatorname{diam} \Omega\} .
$$

Proposition 2.1. Suppose $X$ has a Schauder basis, (2.1) holds, and Hypothesis 1.5 is satisfied. Given $\Omega \subset X$ pseudoconvex open and $u: \Omega \rightarrow \mathbb{R}$, if for each $B \in \mathfrak{B}_{\Omega}$ there are a Banach space $V_{B}$ and $f_{B} \in \mathcal{O}\left(B ; V_{B}\right)$ such that $u(x) \leq\left\|f_{B}(x)\right\|_{V_{B}}$ for all $x \in B$, then there are a Banach space $V$ and $f \in \mathcal{O}(\Omega ; V)$ such that $u(x) \leq\|f(x)\|_{V}$ for all $x \in \Omega$.

Proof of Theorem 1.6. Given $\Omega$, consider the family $\mathfrak{O}$ of open $O \subset \Omega$ for which there are a Banach space $V$ and $f \in \mathcal{O}(O ; V)$ such that $u \leq\|f\|_{V}$ on $O$. To show that $\Omega \in \mathfrak{O}$, we shall argue by contradiction.

Proposition 2.1 can be viewed as a criterion for a pseudoconvex open $O \subset \Omega$ to belong to $\mathfrak{O}$ : if $\mathfrak{B}_{O} \subset \mathfrak{O}$, then $O \in \mathfrak{O}$. Therefore if $\Omega \notin \mathfrak{O}$, there is a ball $B_{1} \notin \mathfrak{O}$ of diameter $d_{1}<\frac{1}{2} \operatorname{diam} \Omega, \bar{B}_{1} \subset \Omega$. Similarly, there is a ball $B_{2} \notin \mathfrak{O}$ of diameter $d_{2}<d_{1} / 2, \bar{B}_{2} \subset B_{1}$, and so on. We obtain a sequence of balls $\bar{B}_{n} \subset B_{n-1}$, of diameter $d_{n} \rightarrow 0, B_{n} \notin \mathfrak{O}$. Let $\{x\}=\bigcap B_{n}$. Since $u$ is bounded above in some neighborhood of $x$, it is bounded above on some $B_{n}$, contradicting $B_{n} \notin \mathfrak{O}$; which contradiction proves the theorem.

Next we turn to the proof of Proposition 2.1.

\section{BALl BUNDLES}

We shall work in the setup of Section 2. In particular, we assume (2.1), and we take $\Omega \subset X$ pseudoconvex. Given $N=1,2, \ldots$, we let $\rho_{N}=$ id $-\pi_{N}, Y_{N}=\rho_{N} X$.

If $A \subset \pi_{N} X \approx \mathbb{C}^{N}$ and $r: A \rightarrow[0, \infty)$ is continuous, the sets

$$
\begin{aligned}
A(r) & =\left\{x \in X: \pi_{N} x \in A,\left\|\rho_{N} x\right\|<r\left(\pi_{N} x\right)\right\}, \\
A[r] & =\left\{x \in X: \pi_{N} x \in A,\left\|\rho_{N} x\right\| \leq r\left(\pi_{N} x\right)\right\},
\end{aligned}
$$

ball bundles over a finite dimensional basis, will be called sets of type (B). Analysis on sets of type (B) is simpler than on general sets; on the other hand a general $\Omega$ can be exhausted by sets of type (B) as follows. Let $d(x)=\min \{1, \operatorname{dist}(x, X \backslash \Omega)\}$ and, given $0<\alpha<1$,

$$
\begin{gathered}
D_{N}\langle\alpha\rangle=\left\{t \in \pi_{N} X:\|t\|<\alpha N, \quad 1<\alpha N d(t)\right\}, \\
\Omega_{N}\langle\alpha\rangle=\left\{x \in X: \pi_{N} x \in D_{N}\langle\alpha\rangle,\left\|\rho_{N} x\right\|<\alpha d\left(\pi_{N} x\right)\right\} .
\end{gathered}
$$

Note that $\Omega_{N}\langle\alpha\rangle \subset \Omega$ is an open set of type (B). These sets are closely related to $\Omega_{N}(\alpha)$ of [L3. Section 7] but are more convenient. Below we shall describe their main properties. 
Proposition 3.1. (a) Each $\Omega_{N}\langle\alpha\rangle$ is pseudoconvex.

(b) $\overline{\Omega_{n}\langle\gamma\rangle} \subset \Omega_{N}\langle\beta\rangle$ if $n \leq N, \gamma \leq \beta / 4$.

(c) For fixed $\gamma$ each $x \in \Omega$ has a neighborhood that is contained in all but finitely $\operatorname{many} \Omega_{N}\langle\gamma\rangle$.

In the proof as well as later we shall use two facts concerning $d$. First, $d(x) \leq$ $d(y)+\|x-y\|$. It follows that if $\|x-y\|<d(x) / 2$, then $d(x) / 2<d(y)<2 d(x)$. Second, $-\log d$ is plurisubharmonic in $\Omega$; for this see [No].

Proof. (a) follows since the inequalities in (3.1) describe sublevel sets of plurisubharmonic functions in $\Omega \cap \pi_{N} X$, resp. $\Omega \cap \pi_{N}^{-1} \Omega$.

(b) Suppose $x \in \overline{\Omega_{n}\langle\gamma\rangle}$. From (2.1)

$$
\begin{gathered}
\left\|\pi_{N} x-\pi_{n} x\right\| \leq\left\|x-\pi_{n} x\right\| \leq \gamma d\left(\pi_{n} x\right)<d\left(\pi_{n} x\right) / 2 \text {, hence } \\
\left\|\pi_{N} x\right\| \leq\left\|\pi_{N} x-\pi_{n} x\right\|+\left\|\pi_{n} x\right\| \leq \gamma+\gamma N<\beta N,
\end{gathered}
$$

and $2 d\left(\pi_{N} x\right)>d\left(\pi_{n} x\right)$. Therefore

$$
\beta N d\left(\pi_{N} x\right)>\beta n d\left(\pi_{n} x\right) / 2>\gamma n d\left(\pi_{n} x\right) \geq 1,
$$

so $\pi_{N} x \in D_{N}\langle\beta\rangle$. Also

$$
\left\|x-\pi_{N} x\right\| \leq\left\|x-\pi_{n} x\right\|+\left\|\pi_{N} x-\pi_{n} x\right\| \leq 2 \gamma d\left(\pi_{n} x\right)<\beta d\left(\pi_{N} x\right),
$$

and indeed $x \in \Omega_{N}\langle\beta\rangle$.

(c) Let $\delta \leq \gamma / 4$. From (3.1) it is clear that $x \in \Omega_{n}\langle\delta\rangle$ for some $n$, so that $\Omega_{n}\langle\delta\rangle$ is a neighborhood of $x$ that is contained in $\Omega_{N}\langle\gamma\rangle$ if $N \geq n$, by part (b).

In studying $\Omega$ through exhaustions $\left\{\Omega_{N}\langle\alpha\rangle\right\}_{N=1}^{\infty}$, one encounters the difficulty that while $\Omega_{N}\langle\alpha\rangle$ are indeed sets of type (B), they are so with respect to the decomposition $X=\pi_{N} X \oplus Y_{N}$, which varies with $N$. To negotiate this inconvenience, we introduce sets $\Omega^{N}\langle\gamma\rangle$ that are of type (B) with respect to the decomposition $X=\pi_{N+1} X \oplus Y_{N+1}$ and at the same time comparable to the $\Omega_{N}\langle\alpha\rangle$ 's. Fix $N$, let $D_{0}=\Omega \cap \pi_{N+1} X$,

$$
\begin{gathered}
p^{N}(s)=\max \left\{\frac{\left\|\pi_{N} s\right\|}{N}, \frac{1}{N d(s)}, \frac{\left\|\rho_{N} s\right\|}{d(s)}\right\}, \quad s \in D_{0}, \\
D^{N}\langle\gamma\rangle=\left\{s \in D_{0}: p^{N}(s)<\gamma\right\}, \quad \gamma \in(0,1), \\
\Omega^{N}\langle\gamma\rangle=\left\{x \in \pi_{N+1}^{-1} D^{N}\langle\gamma\rangle:\left\|\rho_{N+1} x\right\|<\gamma d\left(\pi_{N+1} x\right)\right\} .
\end{gathered}
$$

Proposition 3.2. If $\gamma\left\langle 1 / 4\right.$, then $\Omega_{N}\langle\gamma\rangle \subset \Omega^{N}\langle 4 \gamma\rangle$ and $\Omega^{N}\langle\gamma\rangle \subset \Omega_{N}\langle 4 \gamma\rangle$.

Proof. Suppose $x \in \Omega_{N}\langle\gamma\rangle$. To check $s=\pi_{N+1} x \in D^{N}\langle 4 \gamma\rangle$, using (3.2), estimate

$$
\left\|\rho_{N} \pi_{N+1} x\right\|=\left\|\pi_{N+1} x-\pi_{N} x\right\|<\gamma d\left(\pi_{N} x\right)<d\left(\pi_{N} x\right) / 2,
$$

whence $\left\|\pi_{N+1} x\right\|<\left\|\pi_{N} x\right\|+\gamma<\gamma(N+1)$. Also $d\left(\pi_{N} x\right) \leq 2 d\left(\pi_{N+1} x\right)$ follows, and

$$
2 \gamma N d\left(\pi_{N+1} x\right) \geq \gamma N d\left(\pi_{N} x\right)>1 \text {. }
$$

Finally by (3.6), $\left\|\rho_{N} \pi_{N+1} x\right\|<\gamma d\left(\pi_{N} x\right) \leq 2 \gamma d\left(\pi_{N+1} x\right)$; the upshot is that $\pi_{N+1} x$ $\in D^{N}\langle 4 \gamma\rangle$. Now

$$
\left\|\rho_{N} x\right\|-\left\|\pi_{N+1} x-\pi_{N} x\right\| \leq\left\|\rho_{N+1} x\right\| \leq\left\|\rho_{N} x\right\|+\left\|\pi_{N+1} x-\pi_{N} x\right\|,
$$

and this implies $\left\|\rho_{N+1} x\right\|<2 \gamma d\left(\pi_{N} x\right) \leq 4 \gamma d\left(\pi_{N+1} x\right)$; therefore $x \in \Omega^{N}\langle 4 \gamma\rangle$. 
Conversely, suppose $x \in \Omega^{N}\langle\gamma\rangle$ so that $s=\pi_{N+1} x \in D^{N}\langle\gamma\rangle$. To check $t=$ $\pi_{N} x \in D_{N}\langle 4 \gamma\rangle$, first note that $\left\|\pi_{N} x\right\|=\left\|\pi_{N} \pi_{N+1} x\right\| \leq\left\|\pi_{N+1} x\right\|<\gamma N$ by (2.1), (3.3), (3.4). Next, by (3.3), (3.4)

$$
\left\|\pi_{N} x-\pi_{N+1} x\right\|=\left\|\rho_{N} \pi_{N+1} x\right\|<\gamma d\left(\pi_{N+1} x\right)<d\left(\pi_{N+1} x\right) / 2,
$$

whence $d\left(\pi_{N+1} x\right)<2 d\left(\pi_{N} x\right)$ and

$$
4 \gamma N d\left(\pi_{N} x\right)>\gamma N d\left(\pi_{N+1} x\right)>1,
$$

i.e., $\pi_{N} x \in D_{N}\langle 4 \gamma\rangle$ is proved. Furthermore, (3.7), (3.5), (3.8) imply $\left\|\rho_{N} x\right\|<$ $2 \gamma d\left(\pi_{N+1} x\right) \leq 4 \gamma d\left(\pi_{N} x\right)$, so that indeed $x \in \Omega_{N}\langle 4 \gamma\rangle$.

Next we turn to holomorphic approximation on $\Omega_{N}\langle\alpha\rangle$ :

Theorem 3.3. Assume Hypothesis 1.5 with some $\mu \in(0,1)$. If $\gamma<2^{-6} \mu \alpha$ and $V$ is a Banach space, then any $\psi \in \mathcal{O}\left(\Omega_{N}\langle\alpha\rangle ; V\right)$ can be approximated by $\phi \in \mathcal{O}(\Omega ; V)$, uniformly on $\Omega_{N}\langle\gamma\rangle$.

The proof is based on a general approximation theorem on sets of type (B). Recall that a compact subset $C$ of a complex manifold $M$ is plurisubharmonically convex if for each $x \in M \backslash C$, with some plurisubharmonic function $p$ on $M$, we have $\sup _{C} p<p(x)$. If $M \subset \mathbb{C}^{n}$ is open and pseudoconvex, this is equivalent to $C$ being holomorphically convex in $M$; see $[\underline{H}$, Theorem 4.3.4]. The result we shall presently need is [L3] Theorem 6.1]:

Proposition 3.4. Assume Hypothesis 1.5. Let $D \subset \subset D_{0} \subset \pi_{N+1} X$ be relatively open subsets, $C \subset D$ compact and plurisubharmonically convex in $D_{0}$. Let $r$, $R: \bar{D} \rightarrow(0, \infty)$ be continuous, $r<\mu R$. If $D(R)$ is pseudoconvex, then any $f \in \mathcal{O}(D(R) ; V)$ can be approximated by $g \in \mathcal{O}\left(\pi_{N+1}^{-1} D_{0} ; V\right)$, uniformly on $C[r]$.

Proof of Theorem 3.3. First we show that the function $\psi$ can be approximated by $\phi_{1} \in \mathcal{O}\left(\Omega_{N+1}\langle\alpha\rangle ; V\right)$, uniformly on $\Omega_{N}\langle 4 \gamma\rangle$. To this end we shall apply Proposition 3.4 with $D_{0}=\Omega \cap \pi_{N+1} X$ and with notation as in (3.3)-(3.5), $D=D^{N}\langle\alpha / 4\rangle$, $C=\left\{s \in D_{0}: p^{N}(s) \leq 2^{4} \gamma\right\}, R=\alpha d / 4, r=2^{4} \gamma d<\mu R$. Since $p$ is plurisubharmonic, $C$ is plurisubharmonically convex in $D_{0}$, and $D(R)$ is pseudoconvex by [L3. Proposition 4.1]: the assumptions of Proposition 3.4 are satisfied.

By Proposition 3.2, $\psi$ is holomorphic on $\Omega^{N}\langle\alpha / 4\rangle=D(R) \subset \Omega_{N}\langle\alpha\rangle$. Proposition 3.4 implies it can be approximated by $g \in \mathcal{O}\left(\pi_{N+1}^{-1} D_{0} ; V\right)$, uniformly on $C[r] \supset$ $\Omega^{N}\left\langle 2^{4} \gamma\right\rangle \supset \Omega_{N}\langle 4 \gamma\rangle$. Since $\Omega_{N+1}\langle\alpha\rangle \subset \pi_{N+1}^{-1} D_{0}$, one can take $\phi_{1}=g \mid \Omega_{N+1}\langle\alpha\rangle$.

Therefore, given $\epsilon>0$, one can inductively construct $\phi_{j} \in \mathcal{O}\left(\Omega_{N+j}\langle\alpha\rangle ; V\right)$ such that $\phi_{0}=\psi$ and

$$
\left\|\phi_{j}-\phi_{j-1}\right\|_{V}<2^{-j} \epsilon \quad \text { on } \Omega_{N+j}\langle 4 \gamma\rangle, \quad j \in \mathbb{N} .
$$

Proposition 3.1(c) implies the $\phi_{j}$ converge to some $\phi \in \mathcal{O}(\Omega ; V)$ locally uniformly on $\Omega$. In view of Proposition 3.1(b) $\Omega_{N}\langle\gamma\rangle \subset \Omega_{N+j}\langle 4 \gamma\rangle$, so that by (3.9), $\|\phi-\psi\|_{V}<\epsilon$ on $\Omega_{N}\langle\gamma\rangle$.

\section{The proof of Proposition 2.1}

We shall need a technical result about modifying holomorphic functions on sets of type (B). We continue with the setup of Section 3. Fix $N \in \mathbb{N}$, write $\pi=\pi_{N+1}$, $\rho=$ id $-\pi, Y=\rho X$. 
Lemma 4.1. Suppose $A_{2} \subset \subset A_{3} \subset \subset A_{4}$ are (relatively) open subsets of $\Omega \cap \pi X$, $A_{1} \subset A_{2}$ is compact and plurisubharmonically convex in $A_{4}$. Let $r_{i}: A_{4} \rightarrow(0, \infty)$ be continuous, $1 \leq i \leq 4 ; r_{i}<r_{i+1}$, with $-\log r_{1}$ plurisubharmonic. Assume that $A_{4}\left(r_{4}\right) \subset \Omega$ and any Banach space valued holomorphic function on $A_{4}\left(r_{4}\right)$ can be approximated by holomorphic functions on $\Omega$, uniformly on $A_{3}\left(r_{3}\right)$. Given a Banach space $Z$ and $g \in \mathcal{O}(X ; Z)$, there are a Banach space $W$ and $h \in \mathcal{O}(\Omega ; W)$ such that

(i) $\|h(x)\|_{W} \leq 1$ if $x \in A_{1}\left[r_{1}\right]$, and

(ii) $\|h(x)\|_{W} \geq\|g(x)\|_{Z}$ if $x \in A_{3}\left(r_{3}\right) \backslash A_{2}\left(r_{2}\right)$.

Proof. A special case of the lemma would imply there are a Banach space $V$ and $\phi \in \mathcal{O}(\Omega ; V)$ such that $\|\phi\|_{V} \leq 1 / 2$ on $A_{1}\left[r_{1}\right]$ and $\|\phi\|_{V} \geq 2$ on $A_{3}\left(r_{3}\right) \backslash A_{2}\left(r_{2}\right)$. First we shall construct such $\phi$. Consider the Hartogs sets in $\pi X \times \mathbb{C} \approx \mathbb{C}^{N+2}$ :

$$
\begin{aligned}
H_{1} & =\left\{(s, \lambda) \in A_{1} \times \mathbb{C}:|\lambda| \leq r_{1}(s)\right\}, \\
H_{i} & =\left\{(s, \lambda) \in A_{i} \times \mathbb{C}:|\lambda|<r_{i}(s)\right\}, \quad 2 \leq i \leq 4 .
\end{aligned}
$$

Since $H_{1}$ is plurisubharmonically, hence holomorphically, convex in $H_{4}$, there are finitely many $\psi_{j} \in \mathcal{O}\left(H_{4}\right), j \in J$, such that all $\left|\psi_{j}(z)\right| \leq 1 / 4$ when $z \in H_{1}$ but $\left|\psi_{j}(z)\right| \geq 4$ for some $j \in J$ when $z \in H_{3} \backslash H_{2}$. Let $L$ denote the space of linear forms on $Y$ of norm $\leq 1$ and let $V$ denote the Banach space of bounded functions $L \times J \rightarrow \mathbb{C}$; the norm on $V$ is the sup norm. Define $\psi \in \mathcal{O}\left(A_{4}\left(r_{4}\right) ; V\right)$ by

$$
\psi(x)(l, j)=\psi_{j}(\pi x, l \rho x), \quad(l, j) \in L \times J .
$$

Note that $x \in A_{1}\left[r_{1}\right]$, resp. $x \in A_{i}\left(r_{i}\right), i \geq 2$, precisely when $(\pi x, l \rho x) \in H_{1}$, resp. $H_{i}$ for all $l \in L$. In particular, if $x \in A_{1}\left[r_{1}\right]$,

$$
\|\psi(x)\|_{V}=\sup _{l, j}\left|\psi_{j}(\pi x, l \rho x)\right| \leq 1 / 4 .
$$

On the other hand if $x \in A_{3}\left(r_{3}\right) \backslash A_{2}\left(r_{2}\right)$, then with $l, j$ such that $z=(\pi x, l \rho x) \in$ $H_{3} \backslash H_{2}$ and $\left|\psi_{j}(z)\right| \geq 4$ we have

$$
\|\psi(x)\|_{V} \geq\left|\psi_{j}(\pi x, l \rho x)\right| \geq 4 .
$$

Now take $\phi \in \mathcal{O}(\Omega ; V)$ to be a function that approximates $\psi$ within $1 / 4$ on $A_{3}\left(r_{3}\right)$.

Going back to the general case, since $g$ is bounded on a neighborhood of the compact set $A_{1}$, one can find $q \in \mathbb{N}$ such that

$$
\|g(y)\|_{Z} \leq 2^{q}, \quad \text { if } y \in \pi^{-1} A_{1} \text { and }\|\rho y\| \leq 2^{-q} \max _{A_{1}} r_{1} .
$$

Let $K$ be the space of linear forms on $V$ of norm $\leq 1, \Lambda=\{\lambda \in \mathbb{C}:|\lambda| \leq 1\}$, and let $W$ be the Banach space of bounded functions $K \times \Lambda \rightarrow Z$. The norm on $W$ is again the sup norm. Define $h \in \mathcal{O}(\Omega ; W)$ by

$$
h(x)(k, \lambda)=(k \phi(x))^{q} g\left(\pi x+(\lambda k \phi(x))^{q} \rho x\right), \quad(k, \lambda) \in K \times \Lambda .
$$

If $x \in A_{1}\left[r_{1}\right]$, then $|k \phi(x)| \leq\|\phi(x)\|_{V} \leq 1 / 2$ for all $k \in K$, and (4.1) implies $\|h(x)\|_{W} \leq 2^{-q} \cdot 2^{q}=1$. If $x \in A_{3}\left(r_{3}\right) \backslash A_{2}\left(r_{2}\right)$, choose $k \in K$ so that $k \phi(x)=$ $\|\phi(x)\|_{V} \geq 2 ;$ then

$$
\|h(x)(k, \lambda)\|_{Z} \geq\|g(x)\|_{Z}, \quad \lambda=1 / k \phi(x),
$$

and (ii) follows. 
Proposition 4.2. Assume Hypothesis 1.5, and let $2^{4} \beta<\alpha<2^{-8} \mu$. If $Z$ is a Banach space and $g \in \mathcal{O}(X ; Z)$, then there are a Banach space $W$ and $h \in \mathcal{O}(\Omega ; W)$ such that

(i) $\|h(x)\|_{W} \leq 1$ if $x \in \Omega_{N}\langle\beta\rangle$, and

(ii) $\|h(x)\|_{W} \geq\|g(x)\|_{Z}$ if $x \in \Omega_{N+1}\langle\alpha\rangle \backslash \Omega_{N}\langle\alpha\rangle$.

Proof. Let $\alpha_{3}=4 \alpha$ and fix $\alpha_{4}<1$ so that $2^{6} \alpha_{3}<\mu \alpha_{4}$. Set $D_{0}=\Omega \cap \pi_{N+1} X$, and with the notation in (3.1), (3.3)-(3.5), define bounded sets

$$
A_{1}=\left\{s \in D_{0}: p^{N}(s) \leq 4 \beta\right\}, \quad A_{2}=D^{N}\langle\alpha / 4\rangle, \quad A_{i}=D_{N+1}\left\langle\alpha_{i}\right\rangle, \quad i=3,4,
$$

of which $A_{1}$ is compact and $A_{i}$ is open in $D_{0}, i \geq 2$. Also let $r_{1}=4 \beta d, r_{2}=\alpha d / 4$, $r_{i}=\alpha_{i} d, i=3,4$. We shall apply Lemma 4.1. Clearly $A_{1} \subset A_{2}$ is plurisubharmonically convex in $D_{0}$, hence in $A_{4} \subset D_{0}$. By (3.1), (3.5), and Proposition 3.2

$$
\begin{gathered}
A_{1}\left[r_{4}\right] \supset \Omega^{N}\langle 4 \beta\rangle \supset \Omega_{N}\langle\beta\rangle, \\
A_{2}\left(r_{2}\right)=\Omega^{N}\langle\alpha / 4\rangle \subset \Omega_{N}\langle\alpha\rangle, \quad A_{i}\left(r_{i}\right)=\Omega_{N+1}\left\langle\alpha_{i}\right\rangle, \quad i=3,4 .
\end{gathered}
$$

It follows, again by Proposition 3.2, that $A_{2}\left(r_{2}\right) \subset \Omega_{N}\langle\alpha\rangle$, whence by Proposition 3.1(b), $\overline{A_{2}\left(r_{2}\right)} \subset \Omega_{N+1}\left\langle\alpha_{3}\right\rangle$. Intersecting with $\pi_{N+1} X, \bar{A}_{2} \subset A_{3}$ follows, while $\bar{A}_{3} \subset A_{4}$ is straightforward. The remaining assumptions of Lemma 4.1 are also satisfied, the approximation property a consequence of Theorem 3.3. Therefore the lemma provides an $h \in \mathcal{O}(\Omega, W)$ as needed: $\|h\|_{W} \leq 1$ on $A_{1}\left[r_{1}\right] \supset \Omega_{N}\langle\beta\rangle$ (cf. (4.2)) and $\|h\|_{W} \geq\|g\|_{Z}$ on

$$
A_{3}\left(r_{3}\right) \backslash A_{2}\left(r_{2}\right) \supset \Omega_{N+1}\langle\alpha\rangle \backslash \Omega_{N}\langle\alpha\rangle \quad \text { (cf. (4.3)). }
$$

Proof of Proposition 2.1. Fix $\alpha<2^{-8} \mu$. In the first step for each $N \in \mathbb{N}$ we shall construct a Banach space $Z_{N}$ and $g_{N} \in \mathcal{O}\left(X ; Z_{N}\right)$ such that $u \leq\left\|g_{N}\right\|_{Z_{N}}$ on $\Omega_{N}\langle\alpha\rangle$. We can assume $u \geq 1$ everywhere. Let $A=\overline{\Omega_{N}\langle\alpha\rangle} \cap \pi_{N} X$, a compact set. If $t \in A$, then

$$
\Omega_{N}\langle\alpha\rangle \cap \pi_{N}^{-1} t \subset B(t, \alpha d(t))
$$

hence $t$ has a neighborhood $U \subset \pi_{N} X$ such that $\Omega_{N}\langle\alpha\rangle \cap \pi_{N}^{-1} U \subset B(t, 2 \alpha d(t))$. Therefore

$$
\Omega_{N}\langle\alpha\rangle \subset \bigcup_{t \in T} B(t, 2 \alpha d(t))
$$

with some finite $T \subset A$.

Next verify that $B_{t}=B(t, 2 \alpha d(t) / \mu) \in \mathfrak{B}$. Indeed, $\bar{B}_{t} \subset \Omega$ since $2 \alpha<\mu$, and

$$
2 \operatorname{diam} B_{t}=8 \alpha d(t) / \mu<2 d(t) \leq \operatorname{diam} \Omega .
$$

Thus by assumption there are Banach spaces $V_{t}$ and $f_{t} \in \mathcal{O}\left(B_{t} ; V_{t}\right)$ with $u \leq$ $\left\|f_{t}\right\|_{V_{t}}$ on $B_{t}$. Further, by Hypothesis 1.5 there are $f_{t}^{\prime} \in \mathcal{O}\left(X ; V_{t}\right)$ that satisfy $\left\|2 f_{t}-f_{t}^{\prime}\right\|_{V_{t}}<1$ on $B(t, 2 \alpha d(t))$; whence $u \leq\left\|f_{t}^{\prime}\right\|_{V_{t}}$ there. If $Z_{N}=\bigoplus_{t \in T} V_{t}$, endowed with the sup norm, and $g_{N}=\bigoplus_{t \in T} f_{t}^{\prime} \in \mathcal{O}\left(X, Z_{N}\right)$, then (4.4) implies $u \leq\left\|g_{N}\right\|_{Z_{N}}$ on $\Omega_{N}\langle\alpha\rangle$.

In the second step we fix $\beta<2^{-4} \alpha$ and apply Proposition 4.2 to obtain $W_{N}$ and $h_{N} \in \mathcal{O}\left(\Omega ; W_{N}\right)$ such that $\left\|h_{N}\right\|_{W_{N}} \leq 1$ on $\Omega_{N}\langle\beta\rangle$ but $\left\|h_{N}\right\|_{W_{N}} \geq\left\|g_{N}\right\|_{Z_{N}} \geq u$ on $\Omega_{N+1}\langle\alpha\rangle \backslash \Omega_{N}\langle\alpha\rangle$. Also set $W_{0}=Z_{1}$ and $h_{0}=g_{1}$. Let $V$ be the $l^{\infty}$ sum of the spaces $W_{N}$ :

$$
V=\left\{\left(w_{N}\right)_{N=0}^{\infty}: w_{N} \in W_{N}, \quad \sup _{N}\left\|w_{N}\right\|_{W_{N}}<\infty\right\},
$$


endowed with the norm $\left\|\left(w_{N}\right)\right\|_{V}=\sup \left\|w_{N}\right\|_{W_{N}}$. The properties of $h_{N}$ in conjunction with Proposition 3.1 imply that $f=\bigoplus_{N} h_{N}$ is in $\mathcal{O}(\Omega ; V)$ and indeed $\|f\|_{V} \geq u$.

\section{Applications}

Here we shall prove Theorems 1.2-1.4.

Proof of Theorem 1.2. Given an open neighborhood $G \subset X \times Y$ of $\Omega \times\{0\}$, we have to construct a pseudoconvex neighborhood $P \subset G$. Let

$$
v(x) \geq-\log \operatorname{dist}((x, 0),(X \times Y) \backslash G)
$$

be plurisubharmonic for $x \in \Omega$. Then

$$
P=\left\{(x, y) \in \Omega \times Y: e^{v(x)}\|y\|<1\right\}
$$

will do.

Proof of Theorem 1.3. With $d: \Omega \rightarrow(0,1]$ as in Sections 3 and 4, choose $f \in$ $\mathcal{O}(\Omega ; V)$ satisfying $\|f(x)\|_{V} \geq 1 / d(x), x \in \Omega$. It is immediate that the graph of $f$ is a closed, rectifiable, and split submanifold of $X \times V$ that is biholomorphic to $\Omega$.

The proof of Theorem 1.4 is more involved. The heart of the matter is the following:

Lemma 5.1. Let $M$ be a complex manifold whose topology is induced by a metric. Let $\mathfrak{G}$ be a countable, locally finite open covering of $M$, and suppose that for each $G \in \mathfrak{G}$ we are given a plurisubharmonic $\varphi_{G}: M \rightarrow \mathbb{R}$, which is bounded on bounded subsets of $M$.

(a) If in $M$ plurisubharmonic domination is possible, then there is a (necessarily plurisubharmonic) $\psi: M \rightarrow \mathbb{R}$ such that for $G \in \mathfrak{G}, \psi-\varphi_{G}$ is plurisubharmonic in G.

(b) If each $\varphi_{G}$ is Lipschitz continuous on bounded subsets of $M$ and holomorphic domination is possible in $M$, then $\psi$ above can be chosen locally Lipschitz continuous.

(c) Both in (a) and (b) $\psi$ can be chosen greater than an arbitrarily given function that is locally bounded above.

Proof. (a) Fix positive numbers $\beta_{G}$ so that $\sum_{G \in \mathfrak{G}} \varphi_{G} / \beta_{G}=\varphi$ is uniformly convergent on bounded subsets of $M$; then $\varphi$ is plurisubharmonic and bounded on bounded subsets. Note that the function $b(x)=\max _{G \ni x} \beta_{G}$ is locally bounded on $\Omega$, and choose a continuous function $a \geq b$. Since $\alpha \varphi-\varphi_{G}$ is plurisubharmonic if $\alpha \geq \beta_{G}$, so is $a(x) \varphi-\varphi_{G}$ if $x \in G$.

Next choose a plurisubharmonic $v: M \rightarrow \mathbb{R}$ such that $v \geq \log (1+a)-\varphi$ and set $\psi=e^{v+\varphi}$. If $x \in G \in \mathfrak{G}$, then $\psi-\varphi_{G}$ is plurisubharmonic near $x$. Indeed, let

$$
F(s, t)=e^{s+t}-a(x) t, \quad s, t \in \mathbb{R},
$$

a convex function, increasing with $s$ and $t$ when $e^{s+t} \geq a(x)$. It follows that $\psi-\varphi_{G}=F(v, \varphi)+\left(a(x) \varphi-\varphi_{G}\right)$ is plurisubharmonic in any open set where $e^{v+\varphi} \geq 1+a>a(x)$, in particular near $x$, as needed.

(b) Under the current assumptions, in part (a) the $\beta_{G}$ can be chosen so that $\varphi$ is Lipschitz continuous on bounded subsets, and $v$ can be chosen of the form $v=\|f\|_{V}$ with $f \in \mathcal{O}(M ; V)$; this implies $\psi=e^{v+\varphi}$ is locally Lipschitz continuous. 
(c) This is obvious, because in (a) and (b) $v$ can be chosen to dominate any given function that is locally bounded above.

Proof of Theorem 1.4. (a) Let $\mathfrak{U}$ be a covering of $\Omega$ by balls so that the bundle $\pi: E \rightarrow \Omega$ is trivial over each $U \in \mathfrak{U}$. Lindelöf's theorem implies that $\mathfrak{U}$ can be chosen countable; its elements are of the form

$$
U_{j}=\left\{x \in X: r_{j}-\left\|x-a_{j}\right\|>0\right\}, \quad j=0,1, \ldots
$$

Following $\mathrm{BF}$ p. 883], define $\chi_{0}(x)=r_{0}-\left\|x-a_{0}\right\|$ and

$$
\chi_{j}(x)=\min \left\{r_{j}-\left\|x-a_{j}\right\|, \frac{1}{j}-r_{i}+\left\|x-a_{i}\right\| ; i<j\right\}, \quad j \geq 1 .
$$

One checks that the sets $U_{j}^{\prime}=\left\{x \in \Omega: \chi_{j}(x)>0\right\} \subset U_{j}$ form a locally finite open covering of $\Omega$.

Proposition 5.2. If $\chi_{j}(x)>0$ for some $j$ and $x$, then there is a Lipschitz continuous, plurisubharmonic $\varphi: X \rightarrow \mathbb{R}$ such that $\varphi+\log \chi_{j}$ is plurisubharmonic in a neighborhood of $x$.

Accepting this for the moment, we proceed with the proof of the theorem. For each $j$ choose a trivialization

$$
E \mid U_{j} \ni z \mapsto\left(\pi(z), h_{j}(z)\right) \in U_{j} \times V_{j}
$$

with $\left(V_{j},\|\|_{j}\right)$ a Banach space and $h_{j} \in \mathcal{O}\left(E \mid U_{j} ; V_{j}\right)$ linear on the fibers of $E$. Define a locally Lipschitz continuous metric $\bar{p}$ on $E$ by

$$
\bar{p}=\sup _{j}\left(\pi_{j}^{*} \chi_{j}\right)\left\|h_{j}\right\|_{j},
$$

where $\left(\pi^{*} \chi_{j}\right)\left\|h_{j}\right\|_{j}$ is extended by 0 to $E \backslash E \mid U_{j}$. Since when computing $\bar{p}(z)$ in (5.2) those $j$ 's can be omitted for which $\chi_{j}(\pi z) \leq 0$, the sup there is locally finite. For this reason, and by Proposition 5.2, for each $x \in \Omega$ there are a neighborhood $G \subset \Omega$ and a Lipschitz continuous, plurisubharmonic $\varphi_{G}: \Omega \rightarrow \mathbb{R}$ such that

$$
\pi^{*} \varphi_{G}+\log \bar{p}=\sup _{j}\left\{\pi^{*}\left(\varphi_{G}+\log \chi_{j}\right)+\log \left\|h_{j}\right\|_{j}\right\}
$$

is plurisubharmonic in $E \mid G$. Since $\Omega$ is paracompact, and in view of Lindelöf's theorem, we can assume the sets $G$ form a countable, locally finite covering of $\Omega$.

Lemma 5.1 applies. With $\psi$ provided by the lemma, $p=e^{\pi^{*} \psi} \bar{p}$ is a seminegative metric on $E$, for if $x \in \Omega$, then with an appropriate $G \ni x$

$$
\log p=\pi^{*}\left(\psi-\varphi_{G}\right)+\left(\pi^{*} \varphi_{G}+\log \bar{p}\right)
$$

is plurisubharmonic on $E \mid G$ (cf. (5.3)); hence so is $p$. When holomorphic domination is possible in $\Omega, \psi$ will be locally Lipschitz continuous, and so will $p$. Finally, if a locally bounded metric $m$ is given on $E$, then $m \leq\left(\pi^{*} c\right) \bar{p}$ with some locally bounded $c: \Omega \rightarrow(0, \infty)$, so that if $\psi$ of Lemma 5.1 dominates $\log c$, then $p \geq m$. This takes care of the seminegative part of the theorem.

(b) The semipositive metrics with the required properties can be constructed from duals of seminegative metrics on $E^{*}$, by way of the canonical embedding of $E$ into the second dual $E^{* *}$.

Proof of Proposition 5.2. First consider the family $\mathcal{F}$ of those functions $f: X \rightarrow \mathbb{R}$ that can be written $f=\alpha-\beta$ with $\alpha, \beta: X \rightarrow(0, \infty)$ Lipschitz continuous, $\log \alpha, \log \beta$ plurisubharmonic. This family contains constants and the functions 
$f(x)=\|x-a\|, a \in X$; furthermore, it is closed under the operation of addition, max, and min (of two elements), and multiplication by a constant. For instance, to see that $f_{i} \in \mathcal{F}, i=1,2$, implies $f=\max \left(f_{1}, f_{2}\right) \in \mathcal{F}$, write $f_{i}=\alpha_{i}-\beta_{i}$. Then

$$
\begin{aligned}
f & =\max \left(f_{1}+\beta_{1}+\beta_{2}, f_{2}+\beta_{1}+\beta_{2}\right)-\left(\beta_{1}+\beta_{2}\right) \\
& =\max \left(\alpha_{1}+\beta_{2}, \alpha_{2}+\beta_{1}\right)-\left(\beta_{1}+\beta_{2}\right) .
\end{aligned}
$$

Since both terms on the last line are logarithmically plurisubharmonic (cf. $\mathrm{H}$, Corollary 1.6.8]) and Lipschitz continuous, $f \in \mathcal{F}$.

This shows that each $\chi_{j}$ in (5.1) can be represented as $\chi_{j}=\alpha-\beta$, with $\alpha, \beta$ logarithmically plurisubharmonic and Lipschitz continuous. We claim that the Lipschitz continuous, plurisubharmonic funtion $\varphi=c(\alpha+\beta)$ will do if $c$ is sufficiently large. Indeed, the function

$$
F(s, t)=c\left(e^{s}+e^{t}\right)+\log \left(e^{s}-e^{t}\right)
$$

will then be convex and increasing in both variables in a neighborhood of the point $(s, t)=(\log \alpha(x), \log \beta(x))$. Hence

$$
\varphi+\log \chi_{j}=F(\log \alpha, \log \beta)
$$

is plurisubharmonic in a neighborhood of $x$, as claimed.

\section{REFERENCES}

[A] A. Arroud, Plongement des variétés analytiques complexes de dimension infinie, Thesis, Lille (1983).

[B] E. Bishop, Mappings of partially analytic spaces, Amer. J. Math. 83 (1961), 209-241. MR 23:A1054

[BF] F. Bonic, J. Frampton, Smooth functions on Banach manifolds, J. of Mathematics and Mechanics 15 (1966), 877-898. MR 33:6647

[D] S. Dineen, Bounding subsets of a Banach space, Math. Ann. 192 (1971), 61-70. MR 46:2428

$[\mathrm{H}] \quad$ L. Hörmander, An Introduction to Complex Analysis in Several Variables, 3rd ed., NorthHolland, Amsterdam, 1990. MR 91a:32001

[J1] B. Josefson, Bounding subsets of $l^{\infty}(A)$, J. Math. Pures et Appl. 57 (1978), 397-421. MR 81a:46019

[J2] B. Josefson, Approximations of holomorphic functions in certain Banach spaces, manuscript (2000).

[L1] L. Lempert, The Dolbeault complex in infinite dimensions, I, J. Amer. Math. Soc. 11 (1998), 485-520. MR 99f:58007

[L2] L. Lempert, Approximation of holomorphic functions of infinitely many variables, II, Ann. Inst. Fourier Grenoble 50 (2000), 423-442. MR 2001g:32052

[L3] L. Lempert, The Dolbeault complex in infinite dimensions III, Invent. Math. 142 (2000), 579-603. MR 2002f:32039

[L4] L. Lempert, Vanishing cohomology for holomorphic vector bundles in a Banach setting, Asian J. Math. to appear.

[Ma] P. Mazet, Letter of July 1998.

[Mu] J. Mujica, Complex analysis in Banach spaces, North-Holland, Amsterdam, 1986. MR 88d:46084

[Na] R. Narasimhan, Imbedding of holomorphically complete complex spaces, Amer. J. Math. 82 (1960), 917-934. MR 26:6438

[No] P. Noverraz, Pseudo-convexité, convexité polynomiale et domaines d'holomorphie en dimension infinie, North-Holland, Amsterdam, 1973. MR 50:10814

[P] I. Patyi, On the $\bar{\partial}$ equation in a Banach space, Bull. Soc. Math. France 128 (2000), 391-406. MR 2002e:58010

[R] R. Remmert, Habilitationsschrift, Münster (1958).

[Sn] I. Singer, Bases in Banach spaces I-II, Springer, Berlin, 1981. MR 45:7451; MR 82k:46024 
[Su] Y. T. Siu, Every Stein variety admits a Stein neighborhood, Invent. Math. 38 (1976/77), 89-100. MR 55:8407]

Department of Mathematics, Purdue University, West Lafayette, Indiana 47907

E-mail address: lempert@math.purdue.edu 